\title{
Modification algorithm of NURBS curve interpolation
}

\section{Wan-Jun Zhang ${ }^{1,2,3, a}$, Shan-Ping Gao ${ }^{1, b}$ Su-Jia Zhang ${ }^{1, c}$ \&Feng Zhang ${ }^{2, d}$}

1 Quanzhou Institute of Information Engineering, 36200, China,

2 Lanzhou Industry and Equipment Co. ,Ltd. , Lanzhou 730050, China,

3 School of Mechanical Engineering, Xian Jiao tong University, China741049

Keywords: CAD/Cam;NURBS curve; interpolation algorithm; NC machine tools; simulation .

\begin{abstract}
In order to solve the problems of modification algorithm of NURBS curve interpolation, Such as interpolation time bigger, NURBS curve step error and chord error are not easy changed , and so on. A novel proposed a modification algorithm of NURBS curve interpolation .The algorithm has merits such as higher interpolation position accuracy ,short processing time and so on. In this simulation ,an open five-axis CNC platform based on SIEMENS 840D CNC system is developed for verifying the proposed modification algorithm of NURBS curve interpolation experimentally .The simulation results show that the algorithm is correct; it is consistent with a NURBS curve interpolation requirements.
\end{abstract}

\section{Introduction}

Modern CAD/CAM manufacturing systems, complex shape surfaces for such as aerospace and car models are usually represented by NURBS(Non-Uniform Rational B-Spline). NURBS has become a mathematical tool used in the field CAD/CAM. It has many character[1-3]: NURBS can give a unified mathematical representation for surfaces and curves. But, NURBS curve interpolation has a shortcoming: interpolation time bigger, NURBS curve step error and chord error are not easy changed ,and so on. Lanzhou University of technology researchers [4-6] proposed an NURBS algorithms which based on real-time interpolation and adaptive interpolation. Literature [79] give some NURBS interpolation algorithms, which makes NC programming complicated and interpolation calculate complicated . Shpitalni et al. [9] derived the same interpolation algorithm by using Taylor's expansion. Houng and Yang [10] were given Cubic spline curve interpolator by using Euler algorithm. Lo and Chung[11-12] proposed the error interpolation algorithm which error calculations changed by curve chord.

On the basis of the research above, a algorithm for modification algorithm of NURBS curve interpolation is presented in this paper. Furthermore, this interpolation algorithm through actual processing of simulation are discussed. The simulation results show that the algorithm is correct; it is consistent with a NURBS curve interpolation requirements.

\section{NURBS Interpolator}

Supposed $p(u)$ can be represented NURBS curve. While NURBS [3] are parametrically mathematical definition by the following Eq.(1):

$$
P\left(u_{i+1}\right)=\frac{\sum_{i=0}^{n} \omega_{i} d_{i} N_{i, k}(u)}{\sum_{i=0}^{n} \omega_{i} N_{i, k}(u)}=\left[\begin{array}{lll}
x\left(u_{i+1}\right) & y\left(u_{i+1}\right) & z\left(u_{i+1}\right)
\end{array}\right]=p\left(X_{i+1}, y_{i+1}, Z_{i+1}\right)
$$

Where $u$ is cubic time NURBS curve each parameter, $\mathrm{k}$ the order of NURBS curve.$p_{i}$ is the control points, $\omega_{i}$ is the weight vector , $N_{i, k}(u)$ is the blending function .

According to the parameter $u_{i}$ at $t=t_{i}$ and the first-order, second-order derivate of function $u_{i+1}$ with $t$, so, we can also calculate $\boldsymbol{u}_{i+1}$ at $t=t_{i+1}$ by Taylor's expansion as equation(2)

$$
u_{i+1}=u_{i}+u_{i}^{\prime} \cdot T+\frac{1}{2} u_{i}^{\prime \prime} \cdot T^{2}+0\left(T^{2}\right) \quad\left(T=t_{i+1}-t_{i}\right)
$$


Where, $T$ is the sampling time, H.O.T. stands for the higher order terms in second derivative of Taylor expansion.

The first derivative of Taylor expansion of $u$ with $t$ is then

$$
u_{i}=\left.\frac{d u}{d t}\right|_{t=t_{i}}
$$

The second derivative of Taylor expansion of $u$ with can be calculated as

$$
u_{i}^{\prime \prime}=\left.\frac{d^{2} u}{d t^{2}}\right|_{t=t_{i}} \approx \frac{u_{i}-u_{i-1}+u_{i-2}}{T^{2}} .
$$

Difference equation instead of differential equation.

$$
u_{i+1}=2.5 u_{i}-2 u_{i-1} \cdot T+\frac{1}{2} u_{i-2}
$$

\section{Modification algorithm of NURBS curve interpolation}

3.1Modification algorithm of NURBS curve

From Eq.(1) get Eq.(6):

$$
P\left(\boldsymbol{u}_{i+1}\right)=p\left(\boldsymbol{X}_{i+1}, y_{i+1}, \boldsymbol{Z}_{i+1}\right)=\left[\begin{array}{lll}
x\left(\boldsymbol{u}_{i+1}\right) & y\left(\boldsymbol{u}_{i+1}\right) & z\left(\boldsymbol{u}_{i+1}\right)
\end{array}\right]
$$

The corresponding estimated feed step can be represented as follows:

$$
\Delta l_{i}^{*}=p\left(u_{i+1}\right)-p\left(u_{i}\right)=\sqrt{\left(X_{i+1}-X_{i}\right)^{2}+\left(y_{i+1}-y_{i}\right)^{2}+\left(Z_{i+1}-z_{i}\right)^{2}}
$$

Bow high step length, The define of NURBS curve chord error is as shown in Fig.1. NURBS curve chord error is used interpolation error instead.

$$
\delta_{i}=\rho_{i}-\sqrt{\rho_{i}-\left(\frac{\Delta L}{2}\right)^{2}}
$$

Where $\rho_{i}$ is the radius of NURBS curve curvature at the $p\left(u_{i}\right)$ and $\delta_{i}$ is the distance between $p\left(u_{i}\right)$ and $p\left(u_{i+1}\right)$.

NURBS curve feed speed $v\left(u_{i}\right)$ is

$$
v\left(u_{i}\right)=\left\|\frac{d p\left(u_{i}\right)}{d t}\right\|=\left\|\frac{d p(u)}{d u} \cdot \frac{d u}{d t}\right\|=\left\|\frac{d p(u)}{d u}\right\| \cdot \frac{d u}{d t}
$$

$\Delta L_{i}$ is estimated step which can get Eq.(9)

$$
\Delta L_{i}=v\left(u_{i}\right) \cdot T
$$

Substituting Eq.(9) Eq.(10)into Eq.(8) will yield

$$
\delta_{i}=\rho_{i}-\sqrt{\rho_{i}-\left(\frac{v\left(u_{i}\right) \cdot T}{2}\right)^{2}} \approx \frac{\left(v\left(u_{i}\right) \cdot T\right)^{2}}{8 \rho_{i}}=\frac{\left(\Delta L_{i}\right)^{2}}{8 \rho_{i}}
$$

According to figure 4 , we should calculate $\delta_{i}$.

$$
\delta_{i}=\left|\frac{p\left(u_{i+1}\right)+p\left(u_{i}\right)}{2}-p\left(u_{i}+\frac{u_{i+1}-u_{i}}{2}\right)\right|
$$

From Eq.(8) get Eq.(13):

$$
\Delta L_{i}=\sqrt{{ }^{8} \delta_{i} \cdot \rho_{i}}
$$

Let, $\delta_{i}=\delta_{\max }$, then $\Delta l_{i}^{*}$ stands for Max $\Delta L_{i}$.

$$
\Delta l_{i}^{*}=\sqrt{{ }^{8} \delta_{\text {max }} \cdot \rho_{i}}
$$

From Eq.(13), Eq.(14),we should get Eq.(15) . 


$$
\frac{\Delta l_{i}}{\Delta l_{i}^{*}}=\frac{\sqrt{{ }^{4} \delta_{i}\left(2 \rho_{i}-\delta_{i}\right)}}{\sqrt{{ }^{4} \delta_{\text {max }}\left(2 \rho_{i}-\delta_{\text {max }}\right)}} \approx \frac{\sqrt{{ }^{8} \delta_{i} \cdot \rho_{i}}}{\sqrt{{ }^{8} \delta_{\max } \cdot \rho_{i}}}=\sqrt{\frac{\delta_{i}}{\delta_{\max }}}
$$

We give a modification algorithm of NURBS curve interpolation which is obtained from Eq.(15)by using the following formula:

$$
u_{i+1}=\frac{\Delta l_{i}}{\Delta l_{i}^{*}}+\left(1-\frac{\Delta l_{i}}{\Delta l_{i}^{*}}\right) u_{i}
$$

By substituting Eq.(15) into Eq.(16), modification algorithm of NURBS curve interpolation can be taken.

3.2 Meet every axis control before modificated interpolation formula

$$
\left\{\begin{array}{l}
\Delta p_{i}=p\left(u_{i+1}\right)-p\left(u_{i}\right) \quad, p_{i}\left(l_{i}\right)=p_{i+1} \\
|| \Delta p_{i} \mid=\Delta l_{i} \quad, u=l_{i}, u=0
\end{array}\right.
$$

Then, $p_{i}(u)$ expresses that NURBS should be included adaptively $u$ and $\boldsymbol{a}_{i}$.

$$
p_{i}(u)=a_{1 i}+a_{2 i} u+a_{3 i} u^{2}+a_{4 i} u^{3}+a_{5 i} u^{4}+a_{6 i} u^{5}+\ldots
$$

Let, as is shown in Eq.(19).

$$
\left\{\begin{array}{c}
v_{0}=p_{0} \\
v_{n+2}=p_{n}
\end{array}, p_{i}=\left|p_{i x}, p_{i y}, p_{i z}\right| \quad, i=1, \cdots, n\right.
$$

Reverse calculation control points, as shown in Eq.(20).

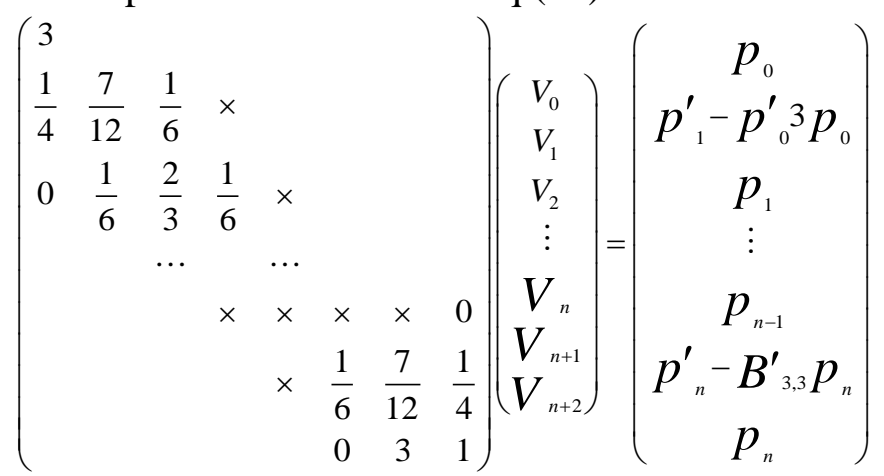

Where, $\times$ denotes Numerical value $\quad \ldots$ denotes 0 .

By using Eq.(17), Eq.(18), Eq.(19) and Eq.(20) the NURBS curve position $\left(x_{i}, y_{i}, z_{i}\right)$ can be calculated by using modification algorithm of NURBS curve interpolation method.

\section{Interpolation algorithm flow chart}

Figure 2 for Flowchart of algorithm. Newton-Rapson iterative interpolation method is explained as follow:

Step1: the input date are from NURBS curve parameters model such as NURBS curve control points, weight vector and so on.

Step2: Calculate Two-orders Taylor's expansion.

Step3:Modifcated interpolation of NURBS curve and calculate control points.

Step4:Get NURBS curve position ${ }^{\left(X_{i}, y_{i}, z_{i}\right)}$ can be calculated by using modification algorithm of NURBS curve interpolation.

Step5: NURBS curve interpolation is finished. 


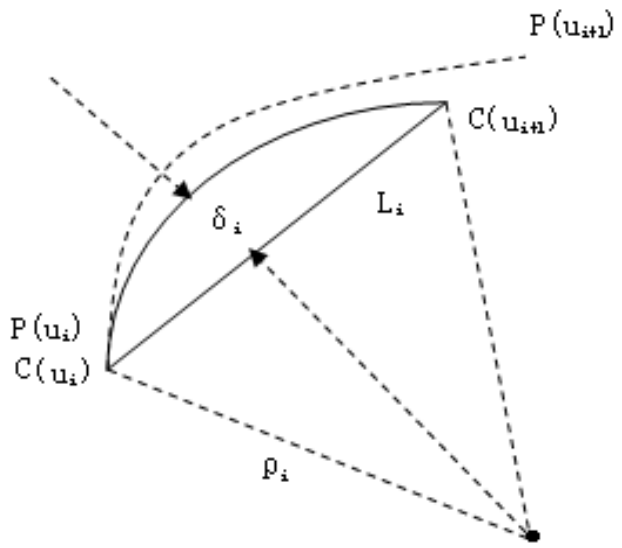

Fig .1: Definition of NURBS curve chord error

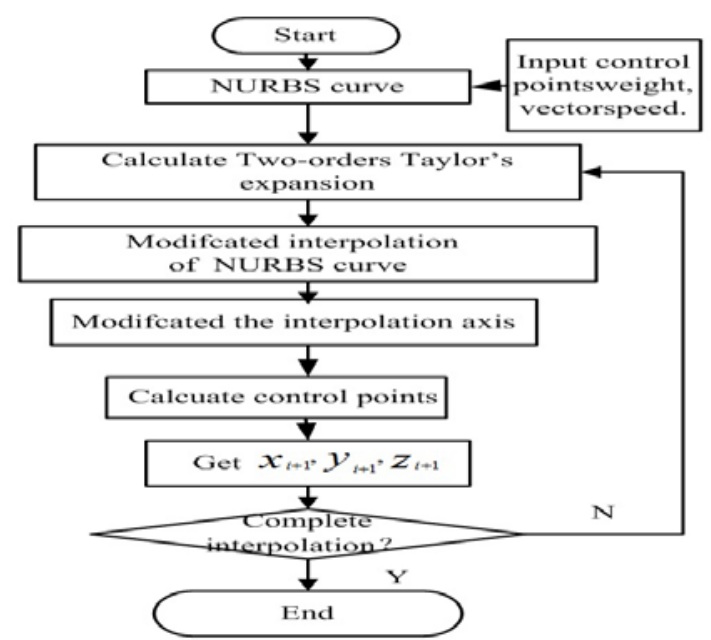

Fig .2: Flowchart of algorithm

\section{Machining simulation}

In this simulation ,An open five-axis CNC platform based on SIEMENS 840D CNC system is developed for verifying the proposed modification algorithm of NURBS curve interpolation experimentally. The experiments of hardware and software structures are as follows:

NC machine tool: machine center.

CPU: PC with AMD Sempron 2,800+2.1Ghz CPU.

Memory:2GB RAM.

Main frequency : $1.44 \mathrm{MHz}$.

Feedback:16 bit digital

Encoder. Dirive system:5-mm scew pitch.

Soft system(OS):32bit Windows 7 .

Machining parameters and dynamics parameters are shown in Table 1.

In the paper, a modification algorithm of NURBS curve interpolation is utilized as an example to the NURBS curve algorithm. The control points ,weight vector, and knot vector of NURBS for the provided example are assigned as follows:

The control pointsare $p_{0}(0,0,0), p_{1}(1,1,2), p_{2}(2,1,2), p_{3}(3,1.5,2), p_{4}(3,2,2.5), p_{5}(3.5,3,3)$ (unit: $\mathrm{mm})$, The weight vector is $\omega_{i}=\left\{\begin{array}{lllll}0 & 0 & 0.405 & 0.0636 & 0.742\end{array}\right\}$, and the knot vector is $u=\left[\begin{array}{lllllllll}1.6 & 1.7 & 0.7 & 1.25 & 0.6 & 0.85 & 1 & 1.2 & 1.2\end{array}\right]$.

The interpolation of NURBS curve parameters are as follows: NURBS curve time in interpolation $T=1 \mathrm{~ms}$; the confined interpolation error is set as $\delta_{\max }=1 \mu \mathrm{m}$; the command interpolation federate is given as $F=300 \mathrm{~mm} / \mathrm{s}$; the allowable acceleration and jerk $J=48000 \mathrm{~mm} / \mathrm{s}^{3}$.

By Using NC machine tool in a modification algorithm of NURBS curve interpolation. The SIEMENS CNC system has a wide and large share in the CNC system market, and they make NC parameters as NC program command NC machine tool, a modification algorithm of NURBS curve interpolation for G-code ,as shown in Tab.1 :

Tab 1 G-code for NURBS interpolation

\begin{tabular}{ccccccccc}
\hline Sequence no & G code & Knot vector & U axis & $\mathrm{x}$ axis & $\mathrm{y}$ axis & $\mathrm{z}$ axis & $\mathrm{W}$ axis & Feedrate \\
\hline N00 & G06.2 & K0.0 & $\mathrm{U} 0$ & $\mathrm{X} 2$ & $\mathrm{Y} 0$ & $\mathrm{Z} 0$ & $\mathrm{~W} 1$ & 1000 \\
& & K0.3 & $\mathrm{U} 0$ & $\mathrm{X}-2$ & $\mathrm{Y} 4$ & $\mathrm{Z} 0$ & $\mathrm{~W} 1 / 3$ & \\
& & $\mathrm{U} 0$ & $\mathrm{X}-2$ & $\mathrm{Y} 0$ & $\mathrm{Z} 0$ & $\mathrm{~W} 1 / 3$ & \\
& & & $\mathrm{U} 1$ & & & & $\mathrm{~W} 1$ & \\
& & & $\mathrm{U} 1$ & & & & & \\
& & & $\mathrm{U} 1$ & & & & &
\end{tabular}

At now, G06.2 is the typical curve interpolation way for G code, such as SIEMENS CNC system. 
Figure 2 for NC machine tool in cubic B-Spline curve interpolation, as shown in Tab.2, Figure 3andFigure 4.
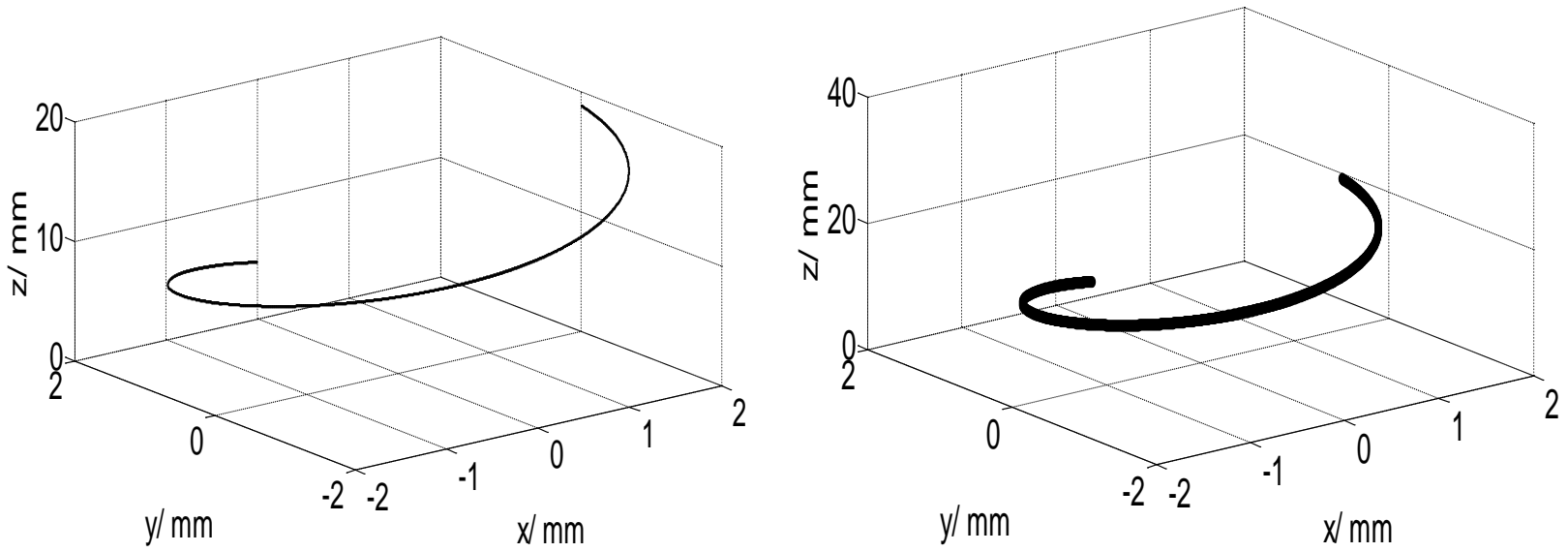

Fig. 3 : NURBS curve interpolation

Fig. 4 : NURBS curve interpolation of modificated algorithm

Tab.2 Table analysis of interpolation of NURBS curve results

\begin{tabular}{lccc}
\hline Parameters algorithms & $\begin{array}{c}\text { Interpolation } \\
\text { time(s) }\end{array}$ & $\begin{array}{c}\text { Max chord } \\
\text { error }(\mathrm{mm})\end{array}$ & $\begin{array}{c}\text { Min chord } \\
\text { error(mm) }\end{array}$ \\
\hline $\begin{array}{l}\text { NURBS curve interpolation } \\
\begin{array}{l}\text { NURBS curve interpolation } \\
\text { of modificated algorithm }\end{array}\end{array}$ & 1.894 & 0.90801 & 0.512 \\
\hline As & 1.55 & 0.79801 & 0.36 \\
\hline
\end{tabular}

As can be seen from Fig. 2, Fig. 4, and table 2, in the process of the interpolation, interpolation time reduced , max chord error too deceased, which meet the expected to interpolation, i.e. to reduce the compensation error and interpolation step chord error. To verify the high efficiency and reliability of this modification algorithm of NURBS curve interpolation are applied in the experiments to make a comparison .It can be seen that modification algorithm of NURBS curve interpolation is feasible and efficent.

\section{Conclusions}

In the paper, a novel on modification algorithm of NURBS curve interpolation is introduced. The algorithm has merits such as higher interpolation position accuracy ,short processing time and so on. In this simulation , an open five-axis CNC platform based on SIEMENS 840D CNC system is developed for verifying the proposed modification algorithm of NURBS curve interpolation experimentally .The simulation results show that the algorithm is correct; it is consistent with a NURBS curve interpolation requirements.

\section{Acknowledgements}

The authors thank the financial supports from National Natural Science Foundation of China(Grant no. 51165024) and Science and Technology Major Project of "High-grade NC Machine Tools and Basic Manufacturing Equipment” (2010ZX040001-181).

\section{References}

[1] Fang Yi-Xiang, Liu Wen-Xue. Based on the geometric properties of the cubic uniform B-Spline curve structure description[J]. Journal of engineering graphics, 2 , pp.96-102, 2006.

[2] Zhang Wan-Jun, Hu Chi-Bing, Zhang Feng , et al . Honing machine motion control card three B spline curve method of interpolation arithmetic for CNC system [J]. Chinese Journal of Manufacturing Technology \& Machine Tool , 8(8), pp.40-43,August 2012. 
[3] Zhang Wan-Jun, HU Chi-bing, WU Zai-xin, et,al. Research on modification algorithm of Three B Spline curve interpolation technology [J].Chinese Journal of Manufacturing Technology \& Machine Tool ,2 pp.141-143,Feburary 2013.

[4] Zhang Wanjun, Zhang Feng , Zhang Guohua. Research on a algorithm of adaptive interpolation for NURBS curve. [J].Applied Mechanics and Materials ,Vol. 687-691, pp.1600-1603, December 2014.

[5] Zhang Wan-Jun, Zhang Feng, Zhang Guohua. Research on modification algorithm of Cubic Bspline curve interpolation technology. [J].Applied Mechanics and Materials, Vol. 687-691, pp.1596-1599, December 2014.

[6] Zhang Wan-Jun, Zhang Feng, Zhang Wan-Liang. Research on a NURBS curve of timing / interrupt interpolation algorithm for CNC system [J].Chinese Journal of Manufacturing Technology \& Machine Tool , 4(4), pp.40-43,April 2015.

[7] Kong Fan-Guo, Hao Shang-Hua, ZhongYan-Zhi. NURBS curve interpolation algorithm to achieve the VC [J]. China new technology and new products, 17, pp.8-9, 2009.

[8] Ye Bo-Sheng,Yang Shu-Zi. CNC system in cubic B-Spline curve interpolation method [J ]. China Mechanical Engineering, 9 ( 3) , pp. 42 - 43, 1998.

[9] Li He-Cheng, Wang Yu-Ping, An interpolation based genetic algorithm for sloving nonlinear bilevel programming problems.Chinese Journal of Computers, 31(6), pp.910-918, June 2008.

[10] Shpitalni M, Koren Y, Lo CC. Realtime curve interpolators. Computer- Aided Design, 26, pp.832-838, 1994.

[11]Huang JT, Yang DCH. A generalized interpolator for command generation of parametric curves in computer controlled machines. Japan/USA Symposium on Flexible Automation,1(1), pp.393-399, January1992.

[12] Lo CC, Chung CY. Curve generation and control for biaxial machine tools. J. CSME , 18, pp.175-182, 1997. 